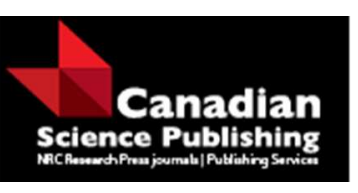

Canadian Journal of Physics

Revue canadienne de physique

\title{
Sandwich Structure Panel Subjected to Thermal Loading using Fractional Order Equation of Motion and Moving Heat Source
}

\begin{tabular}{|r|l|}
\hline Journal: & Canadian Journal of Physics \\
\hline Manuscript ID & cjp-2017-0369.R1 \\
\hline Manuscript Type: & Article \\
\hline Date Submitted by the Author: & 14 -Jul-2017 \\
\hline Complete List of Authors: & $\begin{array}{l}\text { Bassiouny, E.; Prince Sattam in Abdulaziz University, Mathematics; } \\
\text { Fayoum University Faculty of Science, Mathematics } \\
\text { Youssef, Hamdy; Faculty of Engineering, Umm Al-Qura University, P.O. } \\
\text { 5555, Mekkah, Saudi Aribia, Mechanical Engineering Department }\end{array}$ \\
\hline Keyword: & $\begin{array}{l}\text { Sandwich Structure, Moving Heat Source, Fractional Order Equation of } \\
\text { Motion, Fractional Order Strain, Piezoelectric Materials }\end{array}$ \\
\hline $\begin{array}{r}\text { Is the invited manuscript for } \\
\text { consideration in a Special } \\
\text { Issue? : }\end{array}$ & N/A \\
\hline \multicolumn{2}{|c}{} \\
\hline
\end{tabular}

\section{SCHOLARONE ${ }^{\text {m }}$}




\title{
Sandwich Structure Panel Subjected to Thermal Loading using
}

\section{Fractional Order Equation of Motion and Moving Heat Source}

\author{
E. Bassiouny ${ }^{1,2, *}$ and Hamdy M. Youssef ${ }^{3}$ \\ ${ }^{1}$ Department of Mathematics, College of Sciences and Humanitarian Studies, Prince Sattam Bin \\ Abdulaziz University, Saudi Arabia* \\ ${ }^{2}$ Department of Mathematics, Faculty of Sciences, Fayoum University, Fayoum, Egypt \\ ${ }^{3}$ Mechanics Department Faculty of Engineering and Islamic architecture Umm Al-Qura University \\ Saudi Arabia
}

\begin{abstract}
The present work studies the thermoelastic behaviour of a model for a layered thin plate called Sandwich structure subjected to a thermal shock wave in the light of the generalized thermoelasticity theory using fractional order equation of motion in the presence of a moving heat source. The governing equations are solved using Laplace transform. To obtain the different inverse field functions numerically, we used a complex inversion formula of Laplace transform based on Fourier expansion. The effect of different parameters; namely; the speed, the strength of the heat source, fractional order and time on the thermodynamical temperature, stress and on the strain distribution, are discussed and presented graphically. Comparison with previous work in the context of the theory of generalized thermoelasticity shows that the present model is more reliable than the previous. The present model removes the points of discontinuity present in the stress and temperature distribution in the previous model. In the present model we found that the middle layer have affected slight by some of these parameters. The other new results are discussed.
\end{abstract}

Key-Words: Fractional Order Strain, Moving Heat Source, Generalized Thermoelasticity, Fractional Order Equation of Motion, Sandwich Structure, Piezoelectric Materials.

*corresponding author: esambassiouny@ yahoo.com 


\section{Introduction}

Thermoelasticity theory is basically aimed to predicting the thermo-mechanical behaviour of elastic solids, representing elasticity as well as heat conduction theories. The classical uncoupled and coupled thermoelastic theories of Boit [1] and Nowacki [2] have an inherent paradox arising from the assumption that the thermal waves propagate at infinite speed contrary to physical observations. Many authors developed different theories to remove this paradox and to apply the generalized thermoelasticity theory [3][11]. Marine industry widely uses sandwich structures in construction of ship hulls, submarine and hydrofoils. Sandwich and composite structure finds application not only in simple constructions such as buildings, but also in complex constructions of navy and aerospace where the reduction of structural weight is most important. The main advantages of these structures are that they are easy to manufacture and at the same time possess added advantages such as stiffness, weight-strength ratio apart from their thermal insulation properties. Due to the thermal insulation, these structures find place in many industries. Moreover, in different engineering applications such as sensors, actuator and intelligent structure piezoelectric materials, composites and sandwich structures have been used extensively. Many textbooks, handbooks and reviews appeared during the past decade [12]-[14].

In recent years, fractional calculus has been found useful in various physical phenomena in the fields of viscoelasticity, diffusion procedures, relaxation vibrations and in electrochemistry. Various mathematical models of physical process are successfully described by differential equations involving derivatives of fractional (non-integer) order. The fractional order differential operator is non-local. This leads to the dependency of the current state of a system on its previous state. Various definitions and applications of fractional derivatives, have been studied by many researchers [15] - [35]. The famous Riemann-Liouville definition of derivative of fractional order $\beta \in(n-1, n)$ of the function $f(t)$ plays an important role in the theory of fractional derivatives and integrals. One of the most famous form of this definition is given by [15]-[16];

$$
\begin{gathered}
{ }_{R L} D_{t}^{\beta} f(t)=\frac{d^{n}}{d t^{n}}\left[\frac{1}{\Gamma(n-\beta)} \int_{0}^{t}(t-\tau)^{n-\beta-1} f(\tau) d \tau\right] \\
n-1<\beta<n
\end{gathered}
$$

Another definition proposed by Caputo [17]-[19] is;

$$
\begin{gathered}
{ }_{C} D_{t}^{\beta} f(t)=\frac{1}{\Gamma(n-\beta)} \int_{0}^{t}(t-\tau)^{n-\beta-1} \frac{d^{n} f(\tau)}{d \tau^{n}} d \tau \\
n-1<\beta<n
\end{gathered}
$$

where $\Gamma(\beta)$ is the Gamma function. The Caputo fractional derivative has two major advantages. It allows traditional initial and boundary conditions to be included in the formulation of the problem and in addition the derivative of a constant is zero. However, if $f(0)=0$ then, the fractional derivative defined by Caputo and Riemann - Liouville are the same [16].

Kimmich [20] studied time fractional diffusion using Riemann- 
Liouville fractional derivative and considered different values of $\beta$, to express different types of diffusion. For state of weak diffusion $\beta \in(0,1)$; for normal diffusion $\beta=1$, for strong diffusion state $\beta \in(1,2)$ and $\beta=2$ represents ballistic diffusion.

In the present work, we study the thermoelastic properties of a sandwich panel structure subjected to a sudden heating at the ends of the outer layers, considering the theory of generalized thermoelasticity with fractional order strain equation given by Youssef [21] in the presence of a moving heat source. The panel structure consists of three piezoelectric layers. The outer layers are made of Lead Lanthanum Zirconate Titanate $P Z T-5 A$ and the middle layer is made of Barium Titanate $\mathrm{BaTiO}_{3}$.

The piezoelectric material $P Z T-5 A$ is recommended for hydrophones or instrument applications because of its high resistivity at elevated temperatures, high sensitivity, and high time stability [22]. Barium Titanate has been of practical interest for more than 60 years because of its attractive properties: (i) it is chemically and mechanically very stable, (ii) it exhibits ferroelectric properties at and above room temperature, (iii) it can be easily prepared and used in the form of ceramic polycrystalline samples. Due to high dielectric constant and low loss characteristics, Barium Titanate has been used in applications such as capacitor, multilayer capacitors (MLCs), semiconductors and in the piezoelectric devices. The properties of $\mathrm{BaTiO}_{3}$ have been reported in [22] and references there on.

The present model is a new model as it uses a new frac- tional order equation of motion together with the form (3) of the fractional order time derivative of Caputo [17]-[19] to investigate the thermoelastic properties of a sandwich structure panel made of piezoelectric materials subjected to a thermal shock wave on its outer layers in the presence of a moving heat source. The presence of the fractional order operator in the differential equations makes this work more reliable as this parameter allows the equations to take into account the effect of the intermediates as well as the past stages to know the present and next states of the material.

$$
L_{C} D_{t}^{\beta} f(t)=s^{(\beta-n)} L f^{n}(t), \quad n-1<\beta<n,
$$

where $\mathrm{s}$ is a complex parameter related to Laplace transform. The solution of the thermodynamic temperature, stress and strain distribution functions are obtained in the domain of Laplace transform. The inverse field functions are obtained numerically using a complex inversion formula of Laplace transform based on a Fourier expansion and presented graphically. Discussions are also made in the light of the theory of generalized thermoelasticity with fractional order strain equation. Comparison with our previous model [23] in generalized thermoelasticity is also discussed.

\section{One Dimensional Formulation}

For the present one dimensional model, we assume that the structure coincides with the $\mathrm{x}$ - axis having initial temperature $T_{o}$. The free surfaces at $x= \pm 2 h$ are suddenly heated where $h$ is the thickness of the outer layers as shown in fig.1. For this model, it is convenient to assume the follow- 
ing one dimensional form of displacement;

$$
u_{x}=u(x, t), \quad u_{y}=u_{z}=0
$$

where $u_{x}$ is the component of the displacement vector. The generalized thermoelastic one dimensional linearized governing differential equations, in the presence of a moving heat sources according to Youssef [21] are;

The heat equation,

$$
\begin{gathered}
K\left(\frac{\partial^{2} \theta(x, t)}{\partial x^{2}}\right)=\left(\frac{\partial}{\partial t}+\tau_{o} \frac{\partial^{2}}{\partial t^{2}}\right)\left(\rho C_{E} \theta(x, t)+\right. \\
\left.T_{o} \gamma\left(1+\tau^{\beta} D_{t}^{\beta}\right) e(x, t)\right)-\left(1+\tau_{o} \frac{\partial}{\partial t}\right) Q
\end{gathered}
$$

where $K$ is the thermal conductivity, $\tau_{o}$ is the relaxation time, $\rho$ is the density, $C_{E}$ is the specific heat at constant strain, $T_{0}$ is the reference temperature, $\gamma=\alpha_{T}(3 \lambda+2 \mu)$, $\alpha_{T}$ is the thermal linear expansion coefficient, $\theta=T-T_{0}$ is the temperature increment such that $\theta / T_{0} \ll 1, Q$ is the heat source and $e$ is the cubic dilatation.

Equation of motion:

$\rho \frac{\partial^{2} e(x, t)}{\partial t^{2}}=(\lambda+2 \mu)\left(1+\tau^{\beta} D_{t}^{\beta}\right) \frac{\partial^{2} e(x, t)}{\partial x^{2}}-\gamma \frac{\partial^{2} \theta(x, t)}{\partial x^{2}}$

and the constitutive equations can be written in the form;

$$
\sigma(x, t)=\left(1+\tau^{\beta} D_{t}^{\beta}\right)(\lambda+2 \mu) e(x, t)-\gamma \theta(x, t)-\varpi B
$$

and

$$
e(x, t)=\frac{\partial u(x, t)}{\partial x}
$$

The component of the heat flux vector is given by;

$$
q+\tau_{o} \frac{\partial q}{\partial t}=-\kappa \frac{\partial \theta}{\partial x}
$$

where $\varpi$ is the piezoelectric coefficient, $B$ is the component of the electric displacement, $\lambda, \mu$ are Lamé constants and $\sigma$ is the principal stress component.

According to Gauss's law, since there is no free charges inside the medium, we get;

$$
\frac{\partial B}{\partial x}=0
$$

and this gives

$$
B=\text { const }
$$

The medium is traction free and it is subjected to the following boundary conditions at the outer surfaces at the ends $x= \pm 2 h$. The thermal boundary conditions;

$$
\theta(x, t)=f(t) \quad \text { at } \quad x= \pm 2 h
$$

and the mechanical boundary conditions assume the following form;

$$
\sigma(x, t)=0 \quad \text { at } \quad x= \pm 2 h
$$

where $f(t)$ is a prescribed function depends on the time $t$. The medium is assumed to be initially at rest and has reference temperature $T_{o}$ so that the initial conditions become;

$$
\begin{aligned}
& \theta(x, 0)=0, \quad \sigma(x, 0)=0, \\
& \partial \theta(x, 0) / \partial t=0, \quad \partial e(x, 0) / \partial t=0, \quad \partial \sigma(x, 0) / \partial t=0
\end{aligned}
$$

For writing the governing equations (5) - (8) in a dimensionless form, it is convenient to introduce the following 
nondimensional variables Youssef [21];

$$
\begin{aligned}
u^{\prime} & =c_{o} \eta u, t^{\prime}=c_{o}^{2} \eta t, \quad \sigma^{\prime}=\frac{\sigma}{\lambda+2 \mu}, \\
\theta^{\prime} & =\frac{\gamma \theta}{\lambda+2 \mu}, \quad x^{\prime}=c_{o} \eta x, \quad B^{\prime}=\frac{\varpi}{\lambda+2 \mu} B, \\
c_{o}^{2} & =\frac{\lambda+2 \mu}{\rho}, \tau^{\prime}=c_{o}^{2} \eta \tau, \eta=\frac{\rho C_{E}}{\kappa}, q^{\prime}=\frac{q}{\kappa c_{o} \eta T_{o}} \\
\tau_{o}^{\prime} & =c_{o}^{2} \eta \tau_{o}, \tau^{\prime \beta}=c_{o}^{2} \eta \tau^{\beta}, Q^{\prime}=\frac{Q}{\kappa T_{o} c_{o}^{2} \eta^{2}} .
\end{aligned}
$$

where $c_{o}$ is the longitudinal wave speed, $\kappa$ is the coefficient of thermal conductivity and $\eta$ is the thermal viscosity. Using equations (15), after dropping the primes for convenience, in the equations (5) and (6) lead to:

$$
\begin{aligned}
& \frac{\partial^{2} \theta(x, t)}{\partial x^{2}}=\left[\frac{\partial}{\partial t}+\tau_{0} \frac{\partial^{2}}{\partial t^{2}}\right](\theta(x, t)+ \\
& \left.\varepsilon_{1} \xi\left(1+\tau^{\beta} D_{t}^{\beta}\right) e(x, t)\right)-\left(1+\tau_{o} \frac{\partial}{\partial t}\right) Q
\end{aligned}
$$

and

$$
\frac{\partial^{2} e(x, t)}{\partial t^{2}}=\left(1+\tau^{\beta} D_{t}^{\beta}\right) \frac{\partial^{2} e(x, t)}{\partial x^{2}}-\omega \frac{\partial^{2} \theta(x, t)}{\partial x^{2}},
$$

while the constitutive equations (7) and (8) become;

$$
\sigma(x, t)=\left(1+\tau^{\beta} D_{t}^{\beta}\right) e(x, t)-\omega \theta(x, t)-B,
$$

and

$$
e(x, t)=\frac{\partial u(x, t)}{\partial x}
$$

The component of heat flux vector becomes;

$$
q+\tau_{o} \frac{\partial q}{\partial t}=-\frac{\partial \theta}{\partial x}
$$

where $\xi=\frac{\gamma}{\rho C_{E}}, \quad \omega=\frac{\gamma T_{o}}{\lambda+2 \mu}, \varepsilon_{1}=\frac{\gamma^{2} K}{\varrho C_{E}(\lambda+2 \mu)}$ are non dimensional constants. The moving heat source $Q$ is given by the non-dimensional form;

$$
Q=Q_{o} \delta(x-v t)
$$

where $Q_{o}$ and $v$ are the strength and the speed of the heat source respectively and $\delta($.$) is the Dirac delta function.$

The non dimensional system of equations (16)-(19) is the first fractional order thermoelastic model with fractional order strain equation describing the thermoelasticity problem of a sandwich structure panel made of piezoelectric material subjected to a thermal shock wave and in the presence of a moving heat source.

Applying the definition of Laplace transform with the following form of the Laplace transform for the fractional order derivative Caputo (3);

$$
L\left\{{ }_{C} D_{t}^{\beta} f(t)\right\}=s^{(\beta-n)} L\left\{f^{(n)}(t)\right\},
$$

to the equations (16) - (19), we get the following generalized thermoelasticity system of equations with fractional order strain equation [21];

the heat equation;

$$
\begin{gathered}
\frac{\partial^{2} \bar{\theta}(x, s)}{\partial x^{2}}=s\left(s \tau_{o}+1\right)\left[\left(\varepsilon_{1} \xi\left(\tau^{\beta} s^{\beta}+1\right) \bar{e}(x, s)+\right.\right. \\
\bar{\theta}(x, s)]-\varepsilon e^{s x / v}
\end{gathered}
$$

and the equation of motion assumes the form;

$\frac{\partial^{2} \bar{e}(x, s)}{\partial x^{2}}=\frac{1}{1+s^{\beta} \tau^{\beta}}\left[s^{2} \bar{e}(x, s)+(1+s) \omega \frac{\partial^{2} \bar{\theta}(x, s)}{\partial x^{2}}\right]$,

while the transformed constitutive equations take the form;

$$
\begin{gathered}
\bar{\sigma}(x, s)=\left(1+s^{\beta} \tau^{\beta}\right) \bar{e}(x, s)-\omega(1+s) \bar{\theta}(x, s)-\frac{B}{s}, \\
\bar{e}(x, s)=\frac{\partial \bar{u}(x, s)}{\partial x}
\end{gathered}
$$

Combining equations (17) and (18) and applying Laplace 
transform leads to;

$$
\frac{\partial^{2} \bar{\sigma}(x, s)}{\partial x^{2}}=s^{2} \bar{e}(x, s)
$$

After applying the Laplace transform to equation (20), the component of the heat flux vector becomes;

$$
\bar{q}=-\frac{1}{1+s \tau_{o}} \frac{\partial \theta}{\partial x}
$$

where $\varepsilon=\frac{\left(1+s \tau_{o}\right) Q_{o}}{v}$.

\section{Solution in the Laplace Domain}

After some manipulations, the equations (23) - (24) yield,

$$
\left(\frac{d^{4}}{d x^{4}}-a \frac{d^{2}}{d x^{2}}+b\right) \bar{\theta}(x, s)=c e^{-s x / v} .
$$

Similarly we can obtain a fourth order differential equation in the strain given by,

$$
\left(\frac{d^{4}}{d x^{4}}-a \frac{d^{2}}{d x^{2}}+b\right) \bar{e}(x, s)=c e^{-s x / v}
$$

where

$$
\begin{aligned}
a & =s\left(\frac{s}{1+s^{\beta} \tau^{\beta}}+\left(1+s \tau_{o}\right)\left(1+\varepsilon_{1} \xi \omega\right)\right), \\
b & =\frac{s^{3}\left(1+s \tau_{o}\right)}{\left(1+s^{\beta} \tau^{\beta}\right)} \\
c & =\frac{e^{-(s x) / v} Q_{o} s\left(1-v^{2}+s^{\beta} \tau^{\beta}\right)}{v^{3} \varepsilon_{1} \xi\left(1+s^{\beta} \tau^{\beta}\right)\left(1+s \tau_{o}\right)} .
\end{aligned}
$$

Applying the same boundary conditions at both ends of the medium; $x= \pm 2 h$ and from the symmetry of the present structure as shown in figure 1, the most general solution of system of equations (23)-(25) in the domain of Laplace can be obtained;

$$
\bar{\theta}(x, s)=A_{1} \cosh \left(k_{1} x\right)+A_{2} \cosh \left(k_{2} x\right)+\psi e^{-s x / v},
$$

$$
\bar{e}(x, s)=e_{1} \cosh \left(k_{1} x\right)+e_{2} \cosh \left(k_{2} x\right)+M e^{-s x / v},
$$

$\bar{\sigma}(x, s)=\sigma_{1} \cosh \left(k_{1} x\right)+\sigma_{2} \cosh \left(k_{2} x\right)-\frac{D}{s}+L e^{-s x / v}$,

where

$$
\begin{aligned}
M & =\frac{e^{-s x / v}\left[s^{2} \psi+v\left(Q_{o}-s v\left(1+s \tau_{o}\right)\left(1+(1+s) \varepsilon_{1} \xi \omega\right)\right)\right]}{s v^{2} \varepsilon_{1} \xi\left(1+s \tau_{o}\right)}, \\
L & =\frac{e^{-(s x) / v}\left[\left(Q_{o} v\left(1+s \tau_{o}\right)-\left(s v^{2}\left(1+s \tau_{o}\right)-s^{2}\right) \psi\right]\right.}{s v^{2} \varepsilon_{1} \xi\left(1+s^{\beta} \tau^{\beta}\right)\left(1+s \tau_{o}\right)} \\
e_{i} & =A_{i} \frac{\left(k_{i}^{2}-s\left(1+s \tau_{o}\right)\right) \cosh \left(k_{i} x\right)}{s \varepsilon_{1} \xi\left(1+s^{\beta} \tau^{\beta}\right)\left(1+s \tau_{o}\right)} \\
\sigma_{i} & =A_{i} \frac{\left(-k_{i}^{2}+s\left(1+s \tau_{o}\right)\left(1+(1+s) \varepsilon_{1} \xi \omega\right)\right) \cosh \left(k_{i} x\right)}{s \varepsilon_{1} \xi \omega\left(1+s \tau_{o}\right)} \\
\psi & =\frac{c v^{4}}{s^{4}-a s^{2} v^{2}+b v^{4}}, \quad i=1,2
\end{aligned}
$$

and $A_{i}$, are constants depend on s which can be determined by using the boundary conditions and the roots of the characteristic equations;

$$
\begin{aligned}
& k_{1}= \pm\left(\sqrt{a+\sqrt{a^{2}-4 b}}\right) / \sqrt{2}, \\
& k_{2}= \pm\left(\sqrt{a-\sqrt{a^{2}-4 b}}\right) / \sqrt{2}
\end{aligned}
$$

\section{Solution in Different Regions}

Now let us consider a structure made of three piezoelectric layers as shown in figure 1. The outer layers I and III are made of the same materials; namely $\mathrm{BaTiO}_{3}$. We assume that the thickness of Layer I or III is a half of the thickness of layer II. Layer II is made of the piezoelectric material $P Z T-5 A$ and its thickness is a half of the that of the 
panel. The two outer layers are traction free and subjected to a thermal shock at the ends $x= \pm 2 h$, given by, $f(t)=$ $\theta_{o} H(t)$, where $H(t)$ is the Heaviside theta function.

$$
x=-2 h \quad x=-h \quad x=h \quad x=2 h
$$

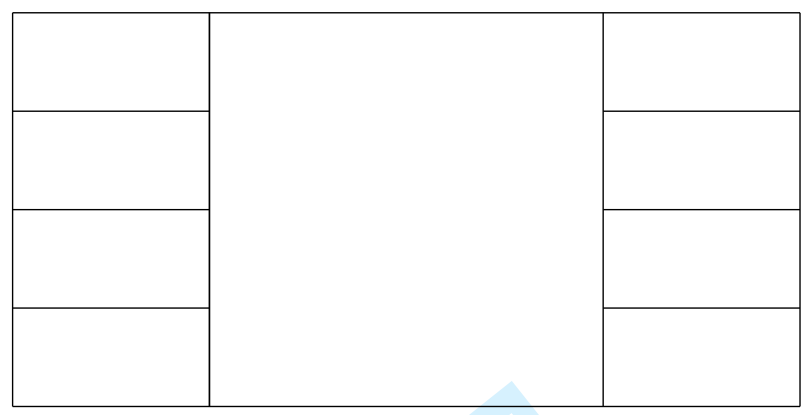

I

II

III

Figure 1: Layers I and III are $\mathrm{BaTiO}_{3}$, layer II is PZT5A

Using the dimensionless variables (15) and by applying the Laplace transform to the boundary and initial conditions (12) - (14) we obtain the following forms of the boundary and initial conditions in the Laplace domain;

$$
\begin{gathered}
\bar{\theta}(2 h, s)=\frac{\theta_{o}}{s}, \quad \bar{\sigma}(2 h, s)=0, \\
\bar{\theta}(-2 h, s)=\frac{\theta_{o}}{s}, \quad \bar{\sigma}(-2 h, s)=0,
\end{gathered}
$$

while the initial conditions (14) becomes,

$$
\bar{\theta}(x, 0)=0, \quad \bar{e}(x, 0)=0, \quad \bar{\sigma}(x, 0)=0 .
$$

By replacing the coefficients $A_{i}, e_{i}, \sigma_{i}, M, L$ and $B$ of the equation (35) with the coefficients which represents the material in thee region I, $A_{i}^{I}, e_{i}^{I}, \sigma_{i}^{I}, M^{I}, L^{I}$ and $B^{I}$ and denoting the roots $k_{i}$ with the roots $k_{i}^{I}$, we get the solution in the region $\mathrm{I},-2 h \leq x \leq-h$, as given below;

$\bar{\theta}^{I}(x, s)=A_{1}^{I} \cosh \left(k_{1}^{I} x\right)+A_{2}^{I} \cosh \left(k_{2}^{I} x\right)+\psi^{I} e^{-s x / v}$

$$
\bar{e}^{I}(x, s)=e_{1}^{I} \cosh \left(k_{1}^{I} x\right)+e_{2}^{I} \cosh \left(k_{2}^{I} x\right)+M^{I} e^{-s x / v}
$$

$$
\bar{\sigma}^{I}(x, s)=\sigma_{1}^{I} \cosh \left(k_{1}^{I} x\right)+\sigma_{2}^{I} \cosh \left(k_{2}^{I} x\right)-\frac{D^{I}}{s}+L^{I} e^{-s x / v},
$$

where the coefficients of the material of the region I, $A_{i}^{I}, e_{i}^{I}, \sigma_{i}^{I}, M^{I}, L^{I}$ take the following forms,

$$
\begin{aligned}
M^{I} & =\frac{e^{-s x / v}\left[s^{2} \psi^{I}+v\left(Q_{o}-s v\left(1+s \tau_{o}^{I}\right)\left(1+(1+s) \varepsilon_{1}^{I} \xi^{I} \omega^{I}\right)\right)\right]}{s v^{2} \varepsilon_{1}^{I} \xi^{I}\left(1+s \tau_{o}^{I}\right)} \\
L^{I} & =\frac{e^{-(s x) / v}\left[\left(Q_{o} v\left(1+s \tau_{o}^{I}\right)-\left(s v^{2}\left(1+s \tau_{o}^{I}\right)-s^{2}\right) \psi^{I}\right]\right.}{s v^{2} \varepsilon_{1}^{I} \xi^{I}\left(1+s^{\beta}\left(\tau^{I}\right)^{\beta}\right)\left(1+s \tau_{o}^{I}\right)} \\
e_{i}^{I} & =\frac{A_{i}^{I}\left(\left(k_{i}^{I}\right)^{2}-s\left(1+s \tau_{o}^{I}\right)\right) \cosh \left(k_{i}^{I} x\right)}{s \varepsilon_{1}^{I} \xi^{I}\left(1+s^{\beta}\left(\tau^{I}\right)^{\beta}\right)\left(1+s \tau_{o}^{I}\right)} \\
\sigma_{i}^{I} & =\frac{A_{i}^{I}\left(\left(-k_{i}^{I}\right)^{2}+s\left(1+s \tau_{o}^{I}\right)\left(1+(1+s) \varepsilon_{1}^{I} \xi^{I} \omega^{I}\right)\right) \cosh \left(k_{i}^{I} x\right)}{\left(1+s \tau_{o}^{I}\right)\left(1+\varepsilon_{1}^{I} \xi^{I} \omega^{I}\right)} \\
\psi^{I} & =\frac{c v^{4}}{s^{4}-a^{I} s^{2} v^{2}+b^{I} v^{4}}
\end{aligned}
$$

To complete the solution in region I, we should determined the coefficients $A_{1}^{I}$ and $A_{2}^{I}$. Using the thermal and mechanical boundary conditions (38), yield;

$$
\begin{aligned}
& A_{1}^{I}=\left(\alpha_{2}\left(\varphi_{0}-e^{2 h s / v} \psi^{I}\right) \operatorname{sech}\left(2 h k_{1}^{I}\right)\right) /\left(\left(k_{2}^{I}\right)^{2}-\left(k_{1}^{I}\right)^{2}\right), \\
& A_{2}^{I}=\left(\alpha_{1}\left(\varphi_{0}-e^{2 h s / v} \psi^{I}\right) \operatorname{sech}\left(2 h k_{2}^{I}\right)\right) /\left(\left(k_{1}^{I}\right)^{2}-\left(k_{2}^{I}\right)^{2}\right) .
\end{aligned}
$$

where $\alpha_{1}=s^{2}-\left(k_{2}^{I}\right)^{2}, \alpha_{2}=s^{2}-\left(k_{1}^{I}\right)^{2}$ and the coefficients $a^{I}, b^{I}$ and $c^{I}$, take the forms (45);

$$
\begin{aligned}
a^{I} & =s\left(\frac{s}{1+s^{\beta}\left(\tau^{I}\right)^{\beta}}+\left(1+s \tau_{o}^{I}\right)\left(1+\varepsilon_{1}^{I} \xi^{I} \omega^{I}\right)\right), \\
b^{I} & =\frac{s^{3}\left(1+s \tau_{o}^{I}\right)}{\left(1+s^{\beta}\left(\tau^{I}\right)^{\beta}\right)} \\
c^{I} & =\frac{e^{-(s x) / v} Q_{o} s\left(1-v^{2}+s^{\beta}\left(\tau^{I}\right)^{\beta}\right)}{v^{3} \varepsilon_{1}^{I} \xi^{I}\left(1+s^{\beta}\left(\tau^{I}\right)^{\beta}\right)\left(1+s \tau_{o}^{I}\right)}
\end{aligned}
$$

Similarly, we obtain the solution in region II in the same form of equations (32)-(34) by replacing the coefficients $A_{i}, e_{i}, \sigma_{i}, M, L$ and $B$ of the equations (35) with the coefficients of the material of the region II, $B_{i}, e_{i}^{I I}, \sigma_{i}^{I I}, M^{I I}, L^{I I}$ 
and $B^{I I}$. The coefficients in region II are given by the

equations (46);

$$
\begin{aligned}
M^{I I} & \left.=e^{-s x / v}\left[s^{2} \psi^{I I}+v\left(Q_{o}-s v\left(1+s \tau_{o}^{I I}\right)\left(1+(1+s) \varepsilon_{1}^{I I} \xi^{I I} \omega^{I I}\right)\right)\right] / s v^{2} \varepsilon_{1}^{I I} \xi^{I I}\left(1+s \tau_{o}^{I I}\right)\right], \\
M^{I I} & =\frac{e^{-s x / v}\left[s^{2} \psi^{I I}+v\left(Q_{o}-s v\left(1+s \tau_{o}^{I I}\right)\left(1+(1+s) \varepsilon_{1}^{I I} \xi^{I I} \omega^{I I}\right)\right)\right]}{s v^{2} \varepsilon_{1}^{I I} \xi^{I I}\left(1+s \tau_{o}^{I I}\right)}, \\
L^{I I} & =\frac{e^{-(s x) / v}\left[Q_{o} v\left(1+s \tau_{o}^{I I}\right)-\left(s v^{2}\left(1+s \tau_{o}^{I I}\right)-s^{2}\right) \psi^{I I}\right]}{s v^{2} \varepsilon_{1}^{I I} \xi^{I I}\left(1+s^{\beta}\left(\tau^{I I}\right)^{\beta}\right)\left(1+s \tau_{o}^{I I}\right)}, \\
e_{i}^{I I} & =\frac{B_{i}\left(p_{i}^{2}-s\left(1+s \tau_{o}^{I I}\right)\right) \cosh \left(p_{i} x\right)}{s \varepsilon_{1}^{I I} \xi^{I I}\left(1+s^{\beta}\left(\tau^{I I}\right)^{\beta}\right)\left(1+s \tau_{o}^{I I}\right)}, \\
\sigma_{i}^{I I} & =\frac{B_{i}\left(-p_{i}^{2}+s\left(1+s \tau_{o}^{I I}\right)\left(1+(1+s) \varepsilon_{1}^{I I} \xi^{I I} \omega^{I I}\right)\right) \cosh \left(p_{i} x\right)}{\left(1+s \tau_{o}^{I I}\right)\left(1+\varepsilon_{1}^{I I} \xi^{I I} \omega^{I I}\right)}, \\
\psi^{I I} & =\frac{c^{I I} v^{4}}{s^{4}-a^{I I} s^{2} v^{2}+b^{I I} v^{4}},
\end{aligned}
$$

where $p_{i}$ are the roots of the corresponding characteristic equation of the equation (36) and they take the forms,

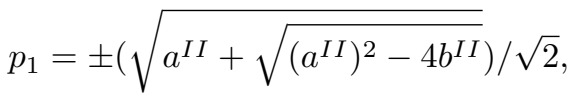

$$
\begin{aligned}
& p_{2}= \pm\left(\sqrt{\left.a^{I I}-\sqrt{\left(a^{I I}\right)^{2}-4 b^{I I}}\right)} / \sqrt{2}\right. \text {. }
\end{aligned}
$$

The coefficients $a^{I I}$ and $b^{I I}$ are the coefficients of the following fourth order differential equation:

$$
\left(\frac{d^{4}}{d x^{4}}-a^{I I} \frac{d^{2}}{d x^{2}}+b^{I I}\right) \bar{\theta}^{I I}(x, s)=c^{I I} e^{-s x / v},
$$

and $B^{I I}$ is the coefficient of the piezoelectric material of the medium occupied the region II. The coefficients $a^{I I}, b^{I I}$ and $c^{I I}$ are given by;

$$
\begin{aligned}
a^{I I} & =s\left(\frac{s}{1+s^{\beta}\left(\tau^{I I}\right)^{\beta}}+\left(1+s \tau_{o}^{I I}\right)\left(1+\varepsilon_{1}^{I I} \xi^{I I} \omega^{I I}\right)\right), \\
b^{I I} & =\frac{s^{3}\left(1+s \tau_{o}^{I I}\right)}{\left(1+s^{\beta}\left(\tau^{I I}\right)^{\beta}\right)} \\
c^{I I} & =\frac{e^{-(s x) / v} Q_{o} s\left(1-v^{2}+s^{\beta}\left(\tau^{I I}\right)^{\beta}\right)}{v^{3} \varepsilon_{1}^{I I} \xi^{I I}\left(1+s^{\beta}\left(\tau^{I I}\right)^{\beta}\right)\left(1+s \tau_{o}^{I I}\right)}
\end{aligned}
$$

To determine the coefficients $B_{1}$ and $B_{2}$, we use the fol- lowing continuity conditions which must satisfied at the boundaries of region II [23];

(i) the thermal boundary conditions: the continuity conditions of the heat flux yields;

$$
\bar{q}^{I}=\bar{q}^{I I} \quad \text { at } \mathrm{x}=\mathrm{-h}, \quad \bar{q}^{I I}=\bar{q}^{I I I} \quad \text { at } \mathrm{x}=\mathrm{h}
$$

Substituting from equation(28) into the equation (50) we get the following form of the thermal continuity conditions;

$$
\begin{aligned}
\frac{\partial \bar{\theta}^{I}}{\partial x} & =\tau_{1} \frac{\partial \bar{\theta}^{I I}}{\partial x} \quad \text { at } \mathrm{x}=\mathrm{-h}, \\
\frac{\partial \bar{\theta}^{I I}}{\partial x} & =\tau_{2} \frac{\partial \bar{\theta}^{I I I}}{\partial x} \quad \text { at } \mathrm{x}=\mathrm{h},
\end{aligned}
$$

where $\tau_{1}=\left(1+s \tau_{o}^{I}\right) /\left(1+s \tau_{o}^{I I}\right)$

and $\tau_{1}=\left(1+s \tau_{o}^{I I}\right) /\left(1+s \tau_{o}^{I I I}\right)$

(ii) the mechanical boundary conditions take the form;

$$
\bar{\sigma}^{I}=\bar{\sigma}^{I I} \text { at } \mathrm{x}=-\mathrm{h}, \quad \bar{\sigma}^{I I}=\bar{\sigma}^{I I I} \text { at } \mathrm{x}=\mathrm{h},
$$

hence the coefficients $B_{1}$ and $B_{2}$ become; 


$$
\begin{aligned}
& B_{1}=\frac{\beta_{2} \cosh \left(h p_{2}\right)\left(v G_{1}+s\left(1-\tau_{1}\right) \psi^{I} e^{-h s / v}\right)-p_{2} \tau_{1} v \operatorname{shin}\left(h p_{2}\right) G_{2}}{\tau_{1} v H}, \\
& B_{2}=\frac{\beta_{1} \cosh \left(h p_{1}\right)\left(v G_{1}+s\left(1-\tau_{1}\right) \psi^{I} e^{-h s / v}\right)-p_{1} \tau_{1} v \operatorname{shin}\left(h p_{1}\right) G_{2}}{\tau_{1} v H}
\end{aligned}
$$

where

$$
\begin{aligned}
G_{1} & =\sum_{i=1}^{2} A_{i}^{I} k_{i}^{I} \operatorname{shin}\left(h k_{i}^{I}\right) \\
G_{2} & =\sum_{i=1}^{2} \alpha_{i} A_{i}^{I} k_{i}^{I} \cosh \left(h k_{i}^{I}\right) \\
H & =p_{1} \beta_{2} \operatorname{shinh} p_{1} \operatorname{coshh} p_{2}-p_{2} \beta_{1} \operatorname{shinh} p_{2} \operatorname{coshh} p_{1}
\end{aligned}
$$

this complete the solution in the region II.

Similarly by replacing the coefficients $A_{i}$ of the equation (32) with the coefficients of the material of the region III, $A_{i}^{I I I}$ and replacing the roots $k_{i}$ with the roots $k_{i}^{I}$, taking into consideration that, the symmetry of the structure in figure 1 and the regions I and III are occupied by the same piezoelectric material; that is they have the same piezoelectric's coefficient $B^{I}$ and the same coefficients $e_{i}^{I}, \sigma_{i}^{I}, M^{I}$ and $L^{I}$, we get the solution in the region III where the coefficients $A_{i}^{I I I}$ take the following forms,

$$
\begin{aligned}
& A_{1}^{I I I}=\left(\gamma_{2}\left(e^{-2 h s / v}-\varphi_{0}\right) \operatorname{sech}\left(2 h k_{1}^{I}\right)\right) /\left(p_{1}^{2}-p_{2}^{2}\right), \\
& A_{2}^{I I I}=\left(\gamma_{1}\left(e^{-2 h s / v}-\varphi_{0}\right) \operatorname{sech}\left(2 h k_{2}^{I}\right)\right) /\left(p_{2}^{2}-p_{1}^{2}\right) .
\end{aligned}
$$

where $\gamma_{1}=s^{2}-p_{2}^{2}$ and $\gamma_{2}=s^{2}-p_{1}^{2}$.

Thus, we have a complete solutions in each region written in the domain of Laplace.

\section{Numerical Inversion of the Laplace}

\section{Transform}

To obtain the solutions of the non-dimensional field functions, the thermodynamical temperature $\theta$, the component of the stress $\sigma$ and the strain distribution $e$ written in each region in the physical domain, we compute numerically the inverse of these field functions by a method based on Fourier expansion technique. In this technique, the original function $f(t)$ of the Laplace transform $\bar{f}(s)$ is approximated by:

$$
\begin{aligned}
f(t)= & \frac{\exp (c t)}{t_{1}}\left[\frac{1}{2} \bar{f}(c)+\right. \\
& \Re\left(\sum_{1}^{N} \bar{f}\left(c+\frac{i k \pi}{t_{1}} \exp \left(\frac{i k \pi}{t_{1}}\right)\right] \quad 0<t_{1}<2 t\right.
\end{aligned}
$$

where $\Re$ is the real part, $i$ is imaginary number unit and $\mathrm{N}$ is a sufficiently large integer representing the number of 
terms in the truncated Fourier series chosen such that:

$$
\exp (c t) \Re\left[\bar{f}\left(c+\frac{i N \pi}{t_{1}}\right) \exp \left(\frac{i N \pi t}{t_{1}}\right)\right] \leq \epsilon_{1}
$$

where $\epsilon_{1}$ is prescribed small positive number that corresponds to the degree of accuracy required. The parameter $c$ is a positive free parameter that must be greater than the real part of all the singularities of $\bar{f}(s)$. The optimal choice of $c$ was obtained according to the criteria described in Honig and Hirdes [24].

\section{Numerical Results and Discussion}

For numerical computation, we use the following physical constants of the piezoelectric materials; Barium Titanate $\mathrm{BaTiO}_{3}$ and Lead Zirconate Titanate $\mathrm{PZT}-5 \mathrm{~A}$ Bassiouny [23]

In figures 2,3 and 5 the solid black lines represent the case when the parameter takes the minimum value; the blue dashed lines represent the case when the parameter takes the maximum value and the red dot-dashed lines represent the case when the value of the parameter lie between the minimum and the maximum value.

We investigate the distributions of the field functions; the thermodynamic temperature $\theta$, the normal stress component $\sigma$ and the strain distribution $e$ with different values of parameters such as the speed $v$ and the strength $Q_{o}$ of the heat source, the fractional order $\beta$ and time $t$. The results were presented in groups of figures, each group illustrates the effect of one of these parameters. Figures 2 show the dependence of the field functions; the thermodynami- cal temperature $\theta$, the stress $\sigma$ and the strain $e$ on the speed of the heat source $v$ at $t=0.3, \tau_{o}=0.2, \tau=0.4$ and $Q_{o}=10^{6}$. Without loss of generalization we may consider any value of the fractional order $\beta \in(0,2]$, for any type of diffusion; we took $\beta=0.5$.

Figure 2(a) shows that the amplitude of the temperature $\theta$ decreases with the increase of the speed of the heat source and it decreases rapidly near the ends of the structure close to the points of application of the loads to the medium. Figure 2(b) illustrates that the stress is compressive near the ends of the medium close to the points of application of the loads and rapidly it turns to be a tensile type. It can be seen that an increase in the value of the speed of the heat source results in an increase in the absolute value of the amplitude of the stress. It is also noticed that the the values of the stress at the outer faces of the structure is equal to zero, in accordance with the boundary conditions at both sides of the boundaries of region $I I I$. It is also noticed that the value of the stress at the boundaries of region II, $x= \pm 3$ does not change in accordance with the continuity condition at these points. Figure 2(c ) illustrate that the strain component resembles the same behaviour like the stress components except at the outer two faces of the structure and at the boundaries of regions $I I$. The strain suffer jumps at $x= \pm 3$.

Figures 3 illustrate the effect of the strength $Q_{o}$ of the heat source on the field functions. Figure 3(a) reflects the profile of the thermodynamical temperature along $x$ - axis. It is noticed that an increase in the value of the strength of 
the heat source results in an increase of the amplitude of the temperature. Significant change of the amplitude of the temperature in all regions can be seen easily. Figure 3(b) represents the behaviour of the stress distribution along the $x$-axis. The absolute value of the amplitude of the stress component decreases with increasing the value of $Q_{o}$. It is also noticed that there are no changes in the amplitude of the stress at the boundaries of the region $I I$ which means that the continuity conditions of the mechanical loads are satisfied at theses boundaries $x= \pm 3$. The behaviour of the strain distribution is presented in Figure 3(c). It is noticed that the amplitude of strain distribution vary inversely with the value of the strength of the heat source. It is also noticed that it suffer discontinuity at the boundary of the core layer $I I$.

Figure 4 shows the dependence of the temperature $\theta$, the stress $\sigma$ and the strain $e$ on the variation of fractional order parameter $\beta$. In figures 4 we consider different values of $\beta$ between 0 and 2 to include all type of diffusion. We notice that the changes in the values of $\beta$ result in changing the absolute value of the amplitude of the field functions. Figure 4(a) shows that the effect of the fractional order parameter is more stronger near the ends points of the outer layers of the structure than the middle layer. We noticed a very slight changes in the magnitude of the temperature in the core layer. Figure 4(b) reflects the variation of the stress with the variation of the fractional order parameter. It is noticed that the absolute value of the magnitude of the stress component decreases with the increasing of the value of the fraction order parameter $\beta$. It is also noticed that the middle layer suffer slight changes in the value of the stress with the variation of the fractional order parameter $\beta$. We notice that the peaks of the distribution curve of the stress occur near the boundaries of each region. The peaks moves slightly towards the core layer of the structure. The equilibrium state occur inside the core layer. Figure 4(c) represents the profile of the strain variation along the $x$-axis. It shows that there are strong changes in the amplitude of the strain components near the ends of the outer layers and it increases as the the fractional order parameter increases. There are two discontinuity points at the points $x= \pm 3$ of the profile of the strain distribution. Figure 5 represents the variation of the profile of the temperature along $x$-axis with variation of the time $t$. The variation of time has significant effects near the points of application of the thermal loads than the middle layer as seen in fig. 5(a). The magnitude of the temperature increases slightly as $t$ increases. It is also noticed that no changes in the amplitude of the temperature in the middle layer as $t$ varies. Figure 5(b) represents the distribution of the stress component with the variation of the time $t$. The stress starts with a compressive type close to the points of application of the thermal loads at $x= \pm 2 h$ and then it changes rapidly at the points $x \simeq \pm 5.8$ into a tensile type. At the points $x \simeq \pm 5.4$ the stress component changes its type again. The absolute value of the magnitude of amplitude of the stress component decreases as the time increases. There are no changes of the stress amplitude through the core layer and the stress component has 
the same amplitude at the boundaries of this region. Figure 5(c) illustrates the effect of variation of time on the distribution of the strain component along the $x$-axis. The absolute value of the magnitude of the amplitude of the strain $e$ decreases as the time increases. Figure 5(c) shows that there are two discontinuity points at $x= \pm 3$.

\section{Conclusion}

The effect of the parameters $v, Q_{o}, \beta$ and $t$ on the field functions $\theta, \sigma$ and strain $e$ are studied and it is noticed that: The fractional order parameter plays an active role in this study; it mainly affects the value of the amplitude of the field functions whatever the type of diffusion. Comparison with previous work [23] shows that the present model in the generalized thermoelasticity with fractional order strain equation removes the points of discontinuity appear in the the temperature distributions in the same model but in the context of generalized thermoelasticity without fractional order. It is also noticed that the thermally induced stress in the two models is of different type near the points of application of the thermal loads. We also noticed that the distributions of the field functions except the strain distributions in the present model are maintained constant inside the core layer in contrary to the previous model in generalized thermoelasticity without fractional order. The strain distributions have two points discontinuity at the boundary of the core layer. In the present model it is noticed that the behaviour of the thermally induced strain with the variation of the speed of the heat source resemble its behaviour with the fractional order as seen in fig 2(c) and 4(c). The distributions of the field functions does in the core layer does not affected by the changes in the value of the fractional order $\beta$ parameters. The thermodynamical temperature $\theta$ behaves in reverse manners with the speed $v$ and strength $Q_{o}$ of the heat source as seen in fig. 2(a) and 3(a). All equilibrium states of all the variation of the field functions with any of the parameters of the present study, $v, Q, \beta$ and time $t$ are maintained inside the core layer. All figures illustrate that the numerical solution of the field functions satisfies the mechanical and the thermal boundary conditions at both sides of the outer faces and satisfy the mechanical and thermal continuity conditions at the boundaries of the core layer.

Acknowledgement This work was supported by the Deanship of Scientific Research at Prince Sattam Bin Abdulaziz University, K.S.A, under the research project No.4119/01/2015. The author is very grateful to Prof. M. A. Kamal, Professor of Computational Fluid Dynamic and Prof Rene George, Professor of pure mathematics Prince Sattam Bin Abdulaziz University, College of Science and Humanitarian Studies for their valuable suggestions for improving the language of this work. The author would like to thank Dr. Raja Gopalan, Assistant Professor at Prince Sattam Bin Abdulaziz University, College of Science and Humanitarian Studies for his technical assistant during this project. 


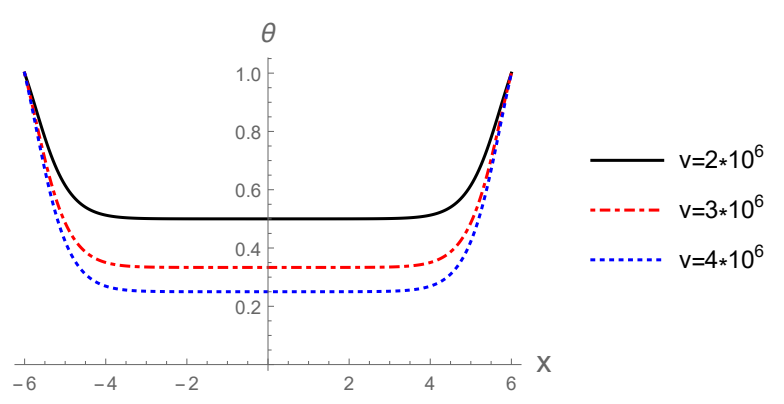

(a) Thermodynamic temperature

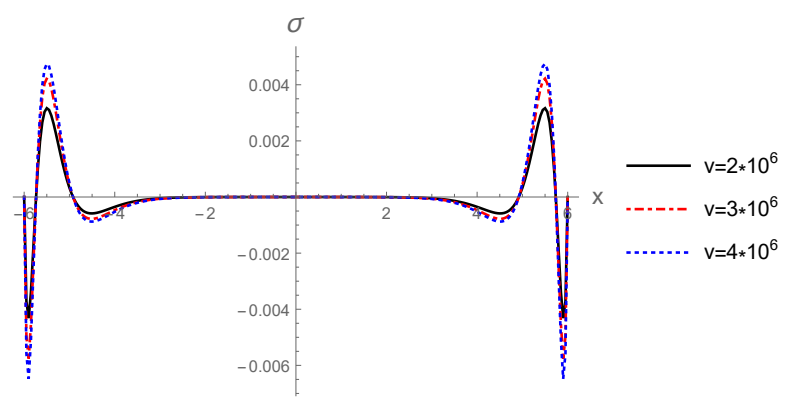

(b) Stress distribution

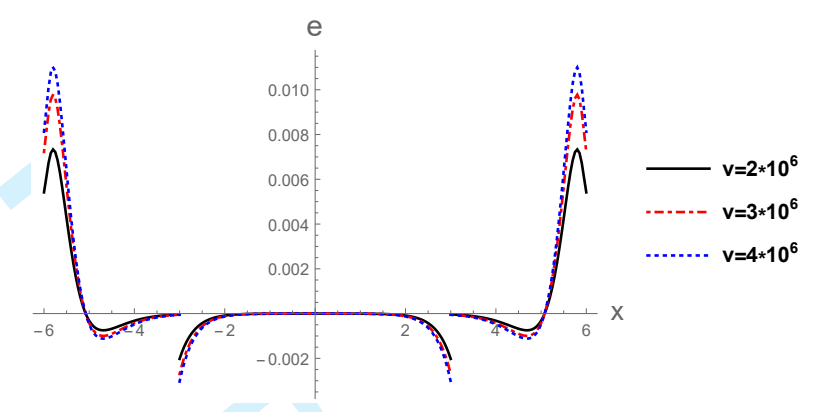

(c) Strain distribution

Figure 2: Effect of the Speed of Heat Source $v$ on the Different filed functions at $t=0.3, \beta=0.5$ and $Q_{o}=10^{6}$

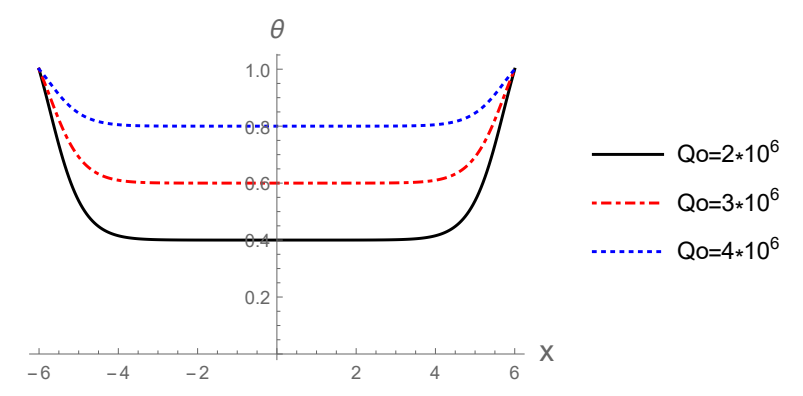

(a) Thermodynamic temperature

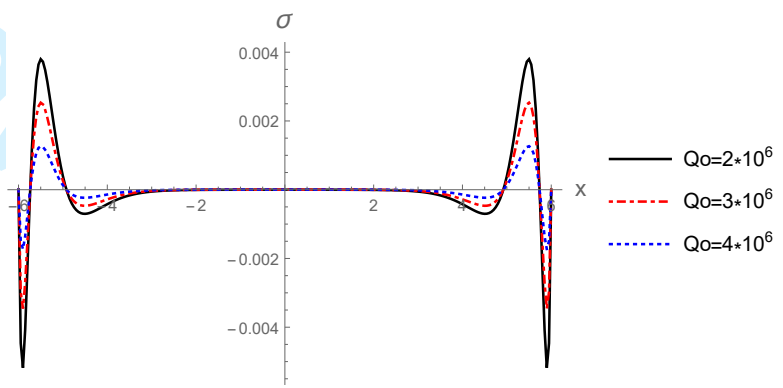

(b) Stress distribution

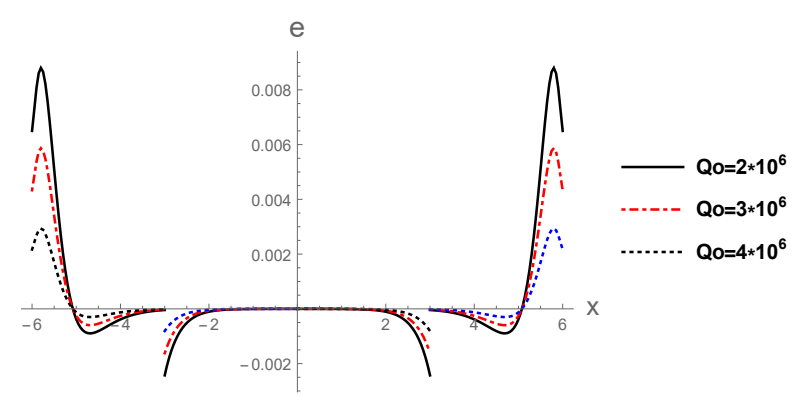

(c) Strain distribution

Figure 3: Effect of Strength of the Heat Source $Q_{o}$ on the Different filed functions at $t=0.3, \beta=0.5, v=3 \times 10^{6}$ 


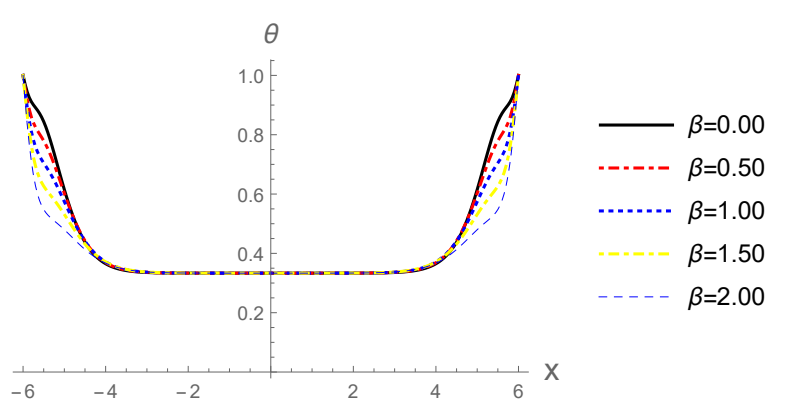

(a) Thermodynamic temperature

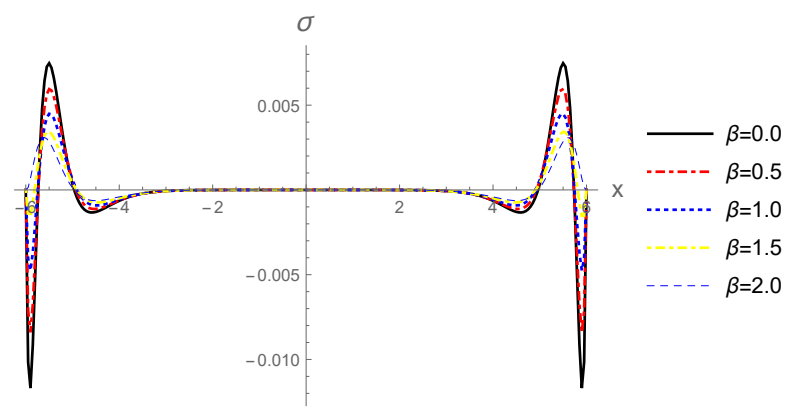

(b) Stress distribution

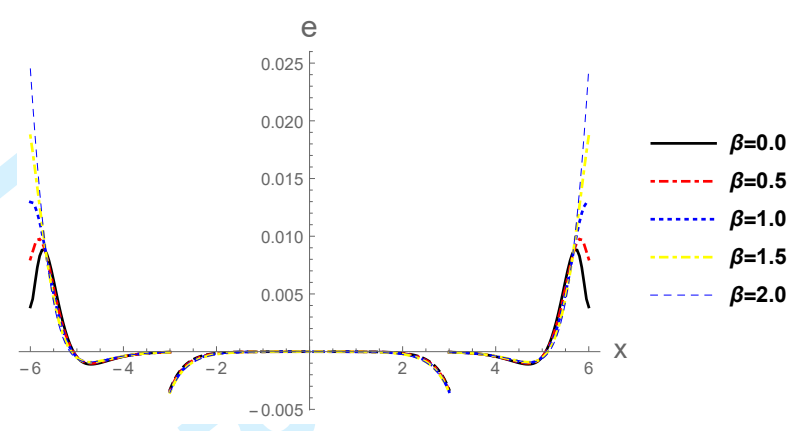

(c) Strain distribution

Figure 4: Effect of parameter $\beta$ on the Different filed functions at $t=0.3, v=3 \times 10^{6}$, and $Q_{o}=10^{6}$

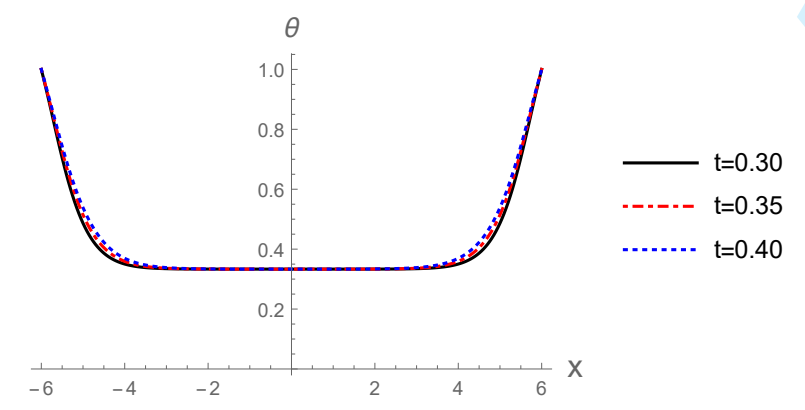

(a) Thermodynamic temperature

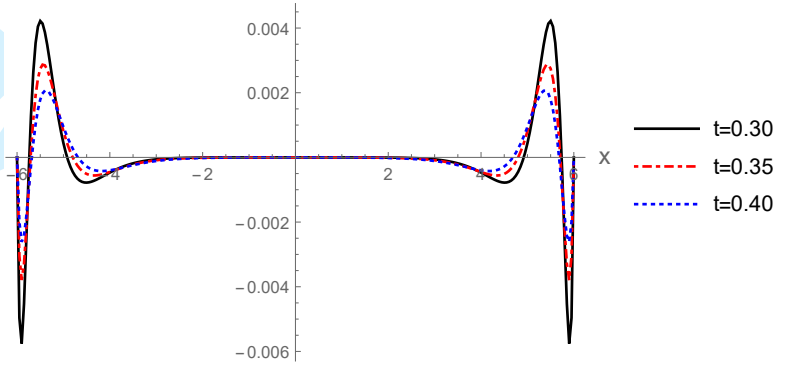

(b) Stress distribution

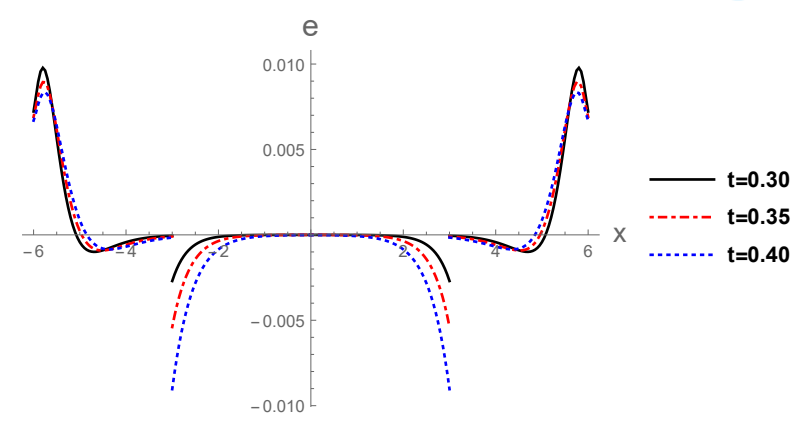

(c) Strain distribution

Figure 5: Effect of time parameter $t$ on the Different filed functions at $\beta=0.5, Q_{o}=10^{6}$ and $v=3 \times 10^{6}$ 


\section{References:}

1. M. A. Biot, The Journal of the acoustical Society of america, 28(2) (1956), 168-178.

2. W. Nowacki, Bulletin L' Academie Polonaise des Science, Serie des Sciences Technology, 14(1966) 129138.

3. H. Lord and Y. Schulman, Journal of the Mechanics and Physics of Solid, 15 (1967) 299-309.

4. I. M. Muller, Archive for Rational Mechanics and Analysis, 41 (1971) 319-332.

5. A. E. Green and K.A. Lindsay, Journal of Elasticity, 2 (1972) 1-7.

6. A. E. Green and N. Laws, Archive for Rational Mechanics and Analysis, 45 (1972), 47-53.

7. E. S. Suhubi and A. C. Eringen, Thermoelastic Solids in Continuum Physics II, (Ch. 2 ed.). (Academic Press, New York, 1975).

8. R. B. Hetnarski and J. Ignaczack,, Journal of Thermal Stresses, 22 (1999), 451-476.

9. R. B. Hetnarski and M. R. Eslami, Thermal StressesAdvanced Theory and Applications,(Springer, Netherlands, Netherlands, 2008).

10. L. Wang, Int. J. Heat and Mass Transfer, 43 (2000), 365-373.

11. M.I.A. Othman, Can. J. Phys., 81(2000), 14031428, (2003).
12. J. HOHE, and L. LIBRESCU, Mechanics of Advanced Materials and Structures, 11(4-5) (2004) 395-424.

13. J.R. Vinson, Appl. Mech. Rev., 54 (2001), 201214.

14. L. Librescu, and T. Hause, Compos. Struct., 48(2000), 117.

15. Atangana, A. and Secer, A., 2013, April. A note on fractional order derivatives and table of fractional derivatives of some special functions. In Abstract and Applied Analysis (Vol. 2013). Hindawi Publishing Corporation.

16. De Oliveira, E.C. and JA, T.M., A review of definitions for fractional derivatives and integral. Mathematical Problems in Engineering, 2014.

17. M. Caputo, Linear model of dissipation whose $\mathrm{Q}$ is always frequency independent-II, Geophysical Journal of the Royal Astronomical Society, 13 (1967), 529539.

18. M. Caputo. Elasticita e Dissipazione. Zanichelli, Bologna, 1969.

19. M. Caputo, Vibrations of an infinite viscoelastic layer with a dissipative memory, J. Acous. Soc. Am., 56 (1974), 897-904.

20. R. Kimmich, Strange Kinetics, porous media, and NMR, J. Chem. Phys., 284 (2002), 243-25.

21. H. M. Youssef, Theory of generalized thermoelasticity 
with fractional order strain, Journal of Vibration and Control, (2015), 1-18.

22. M.M. Vijatovi, J.D. Bobi, B. D. Stojanovi, Science of Sintering, 40, (2008), 155-165.

23. E. Bassiouny, Thermo-elastic behavior of thin sandwich panel made of piezoelectric layers, Appl. Math.and Comp, 218, (2012), 10009-10021.

24. G. Honig and U. Hirdes, J. Comp. Appl. Math., 10, (1984), 113-132.

25. M.I.A.Othman, N. Sarkar and S.Y. Atwa, Effect of fractional order parameter on plane waves of generalized magneto-thermoelastic diffusion with reference temperature-dependent elastic medium, Computer and Math. with. Appl., 65 (7),(2013), 1103-1118.

26. Fujita, Y. Integro differential equation which interpolates the heat equation and wave equation: I. shape Osaka Journal of Mathematics, 27 (1990), 309-321.

27. Fujita, Y. Integro differential equation which interpolates the heat equation and wave equation II., shape Osaka Journal of Mathematics, 27 (1990), 797-804.

28. Miller, K., Ross, B., An Introduction to the Fractional Calculus and Fractional Differential Equations. John Wiley and Sons, Inc., New York, 1993.

29. H. Youssef, Theory of fractional order generalized thermoelasticity, J. Heat Transfer, 132(2010), 1-7.
30. Y. Z. Povstenko, Theories of thermal stresses based on space-time-fractional telegraph equations, Computers and Mathematics with Applications, 64, (2012), 33213328.

31. I. Podlubny, Fractional Differential Equations, Academic Press, New York, 1999.

32. L., Liu, L., Zheng, F., Liu and X., Zhang, Heat conduction with fractional CattaneoChristov upperconvective derivative flux model. International Journal of Thermal Sciences, (2017),112, 421-426.

33. L., Liu L., Zheng, F., Liu and X., Zhang, An improved heat conduction model with space Riesz fractional Cattaneo-Christov flux, Int. J. Heat Mass Transfer, 103(2016), 1191-1197.

34. J., Li, L.,Zheng and L.,Liu MHD viscoelastic flow and heat transfer over a vertical stretching sheet with Cattaneo-Christov heat flux effects, Journal of molecular liquids, 221 (2016) 19-25.

35. S., Han, L., Zheng, C., Li and X., Zhang, Coupled flow and heat transfer in viscoelastic fluid with CattaneoChristov heat flux model, Applied Mathematics Letters, 38(2014) 87-93. 